\title{
Gold(III) Oxide and Oxychloride
}

\author{
Peter G. Jones, Horst Rumpel, George M. Sheldrick and Einhard Schwarzmann
}

Anorganisch-Chemisches Institut der Universität Göttingen, Göttingen, Federal Republic of Germany

Of all metals, gold shows the least tendency to form simple compounds with oxygen; anhydrous gold(III) oxide was until recently unknown. We have successfully prepared single crystals of gold(III) oxide and gold(III) oxychloride large enough to permit structure determination by X-ray crystallography.

Both preparations $(1,2)$ involve the heating in sealed quartz tubes of amorphous hydrated gold(III) oxide with perchloric acid and an alkali metal perchlorate - temperatures and pressures of the order of $250^{\circ} \mathrm{C}$ and $30 \mathrm{MPa}$ are required. Small variations of reagent ratios, temperature and pressure result in different ratios of products and different crystal sizes.

$\mathrm{X}$-ray diffraction data for both compounds have been collected on an automated diffractometer, and both structures have been elucidated $(3,4)$.

Gold(III) oxide exhibits the usual square planar coordination of the metal atom as shown here. The asymmetric unit (smallest unique group of atoms) consists of one gold and two oxygen atoms, one oxygen atom lying on a crystallographic two-fold axis. This oxygen atom $(\mathrm{O} 2$ in the diagram) is co-ordinated to two gold atoms, the other, $\mathrm{Ol}$, is co-ordinated to three gold atoms. This is reflected in the stronger (shorter) Au-O2 bond. A trans effect is observed, the Au-O1 bond trans to $\mathrm{O} 2$ being longest. The extended polymeric structure is best seen as a stereoview (3).

The structure of gold(III) oxychloride is also polymeric, with square planar co-ordination at the metal atom (by three oxygens and one chlorine); it also shows a trans effect, the $\mathrm{AuO}$ bond trans to $\mathrm{Cl}$ being longest.

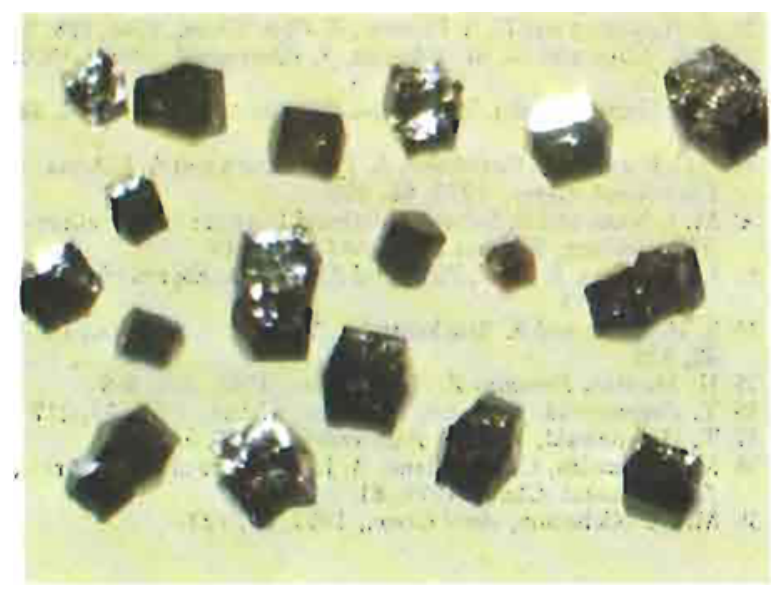

Crystals of $\mathrm{Au}_{2} \mathrm{O}_{3}$ (maximum size $\left.0.2 \mathrm{~mm}\right)$

\section{References}

1 E. Schwarzmann, J. Mohn and H. Rumpel, Z. Naturforsch., 1976, 31b, 135

2 'Handbuch der Präparativen Anorganischen Chemie', Vol. 2 edited by G. Bauer, Ferdinand Enke Verlag, Stuttgart, 1978, p. 1016

3 P. G. Jones, H. Rumpel, E. Schwarzmann, G. M. Sheldrick and H. Paulus, Acta Crystallogr., 1979, B35, 1435-1437

4 P. G. Jones, H. Rumpel, E. Schwarzmann and G. M. Sheldrick, Acta Crystallogr., 1979, B35, 2380-2381

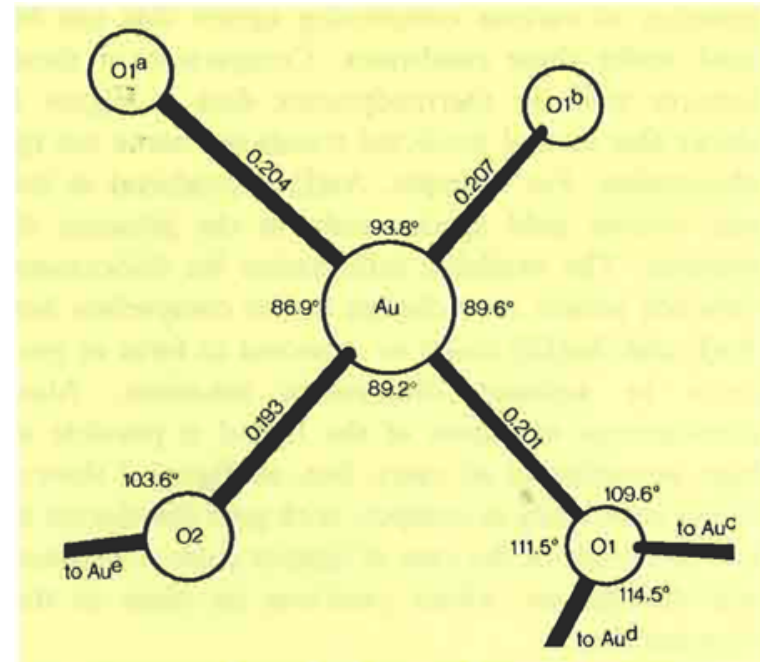

The square planar $\mathrm{AuO}_{4}$ unit in $\mathrm{Au}_{2} \mathrm{O}_{3}$, showing unique bond lengths in $\mathrm{nm}$ and angles in degrees. The superscripts refer to symmetry equivalent atoms

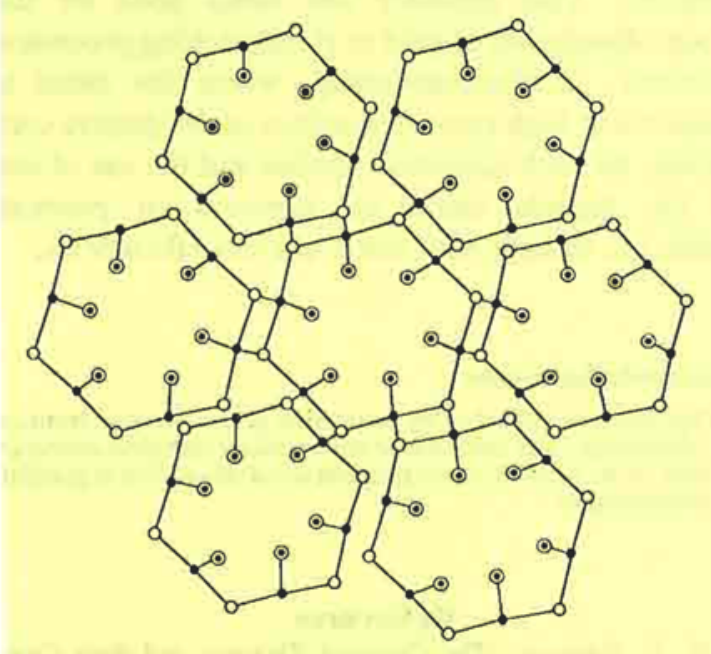

The extended structure of AuOCI. Oxygen, chlorine and gold atoms are represented by open, dotted and filled circles respectively. The structure consists of linked hexameric rings; the gold atoms lie in a plane with oxygen atoms $0.029 \mathrm{~nm}$ and chlorine atoms $0.193 \mathrm{~nm}$ alternatively above and below this plane 